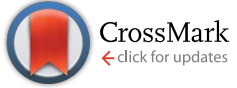

Cite this: Soft Matter, 2015, 11, 303

\title{
From molecular modelling to photophysics of neutral oligo- and polyfluorenes incorporated into phospholipid bilayers $\uparrow$
}

\author{
M. J. Tapia, ${ }^{\text {aa }}$ M. Monteserín, ${ }^{a}$ H. D. Burrows, ${ }^{\text {b }}$ J. A. S. Almeida, ${ }^{b}$ A. A. C. C. Pais, ${ }^{b}$ \\ J. Pina, ${ }^{\mathrm{b}}$ J. S. Seixas de Melo, ${ }^{\mathrm{b}}$ S. Jarmelo ${ }^{\mathrm{b}}$ and J. Estelrich ${ }^{\mathrm{c}}$
}

\begin{abstract}
The combination of various experimental techniques with theoretical simulations has allowed elucidation of the mode of incorporation of fluorene based derivatives into phospholipid bilayers. Molecular dynamics (MD) simulations on a fully hydrated 1,2-dimyristoyl-sn-glycero-3-phosphatidylcholine (DMPC) bilayer, with benzene (B), biphenyl (BP), fluorene (F) and tri-(9,9-di-n-octylfluorenyl-2,7-diyl), TF, have provided insights into the topography of these molecules when they are present in the phospholipid bilayer, and suggest marked differences between the behavior of the small molecules and the oligomer. Further information on the interaction of neutral fluorenes within the phospholipid bilayer was obtained by an infrared (IR) spectroscopic study of films of DMPC and of the phospholipid with PFO deuterated specifically on its alkyl chains (DMPC-PFO- $d_{34}$ ). This was complemented by measurements of the effect of F, TF and two neutral polymers: polyfluorene poly(9,9-di-n-octylfluorenyl-2,7-diyl), PFO, and poly(9,9-di-n-dodecylfluorenyl-2,7-diyl), PFD, on the phospholipid phase transition temperature using differential scanning calorimetry (DSC). Changes in liposome size upon addition of $F$ and PFO were followed by dynamic light scattering. In addition, the spectroscopic properties of F, TF, PFO and PFD solubilised in DMPC liposomes (absorption, steady-state and time-resolved fluorescence) were compared with those of the same probes in typical organic solvents (chloroform, cyclohexane and ethanol). Combining the insight from MD simulations with the results at the molecular level from the various experimental techniques suggests that while the small molecules have a tendency to be located in the phospholipid head group region, the polymers are incorporated within the lipid bilayers, with the backbone predominantly orthogonal to the phospholipid alkyl chains and with interdigitation of them and the PFO alkyl chains.
\end{abstract}

Received 25th September 2014 Accepted 5th November 2014

DOI: $10.1039 / c 4 s m 02145 b$ phase transition, which depends on the lipid alkyl chain length. ${ }^{1}$ In the process of liposome formation, they can entrap both water and lipid soluble compounds, while polyelectrolytes can be adsorbed on their surface through electrostatic interactions. ${ }^{1}$ In addition, liposomes, which typically have sizes on the hundred nanometer scale, can be considered as good carriers for various molecules, and can solubilize neutral compounds, ${ }^{2}$ including polymers, ${ }^{3-5}$ to form aqueous dispersions. ${ }^{5,6}$ The variety of interactions makes liposomes interesting carriers of drugs for therapeutic applications, ${ }^{1,7-9}$ including photodynamic therapy. ${ }^{10}$ In addition, their structural relationship to cell membranes makes liposomes useful models for understanding membrane biophysics, including the way guest molecules are incorporated into phospholipid bilayers. ${ }^{1}$ For example, this can allow us to gain an insight into the interaction of polycyclic aromatic hydrocarbons with membranes to understand how such pollutants penetrate and interact with human skin. The use of fluorescent aromatic compounds, such as fluorene, allows the sensitivity of methods such as steady-state and timeresolved fluorescence, and fluorescence anisotropy to probe the 
interaction between these compounds and the phospholipid bilayer and its effect on membrane properties, ${ }^{\mathbf{1 1 - 1 3}}$ complementing the information obtained with techniques such as differential scanning calorimetry (DSC) on changes in the phospholipid gel-to-liquid phase transitions. ${ }^{2}$ In the last few decades of the twentieth century, discrete fluorescent molecules such as perylene, ${ }^{12}$ xanthene dyes (fluorescein, eosin, Texas Red and rhodamine) ${ }^{13}$ diphenylhexatriene, ${ }^{\mathbf{1 4 , 1 5}}$ trans-parinaric acid ${ }^{\mathbf{1 6}}$ or fluorenyl- ${ }^{17,18}$ and 9-anthroyloxyl- ${ }^{19}$ fatty acids were used and are still widely used ${ }^{20}$ to label liposomes. In the first two decades of the twenty-first century the focus has been extended to the incorporation of conjugated polymers into liposomes..$^{3,4,21-25}$ As Nobel Prize winner Alan Heeger pointed out in a recently published review: "conducting polymers exhibit the electrical and optical properties of metals or semiconductors but with the mechanical and processing advantages of polymer". ${ }^{26}$ The development of conjugated polymers has advanced a long way since the first generation of polyacetylenes, through the second generation of the luminescent, soluble poly(alkylthiophenes) or poly( $p$-phenylenevinylenes) up to the current generation, having donor-acceptor co-polymer repeat units, such as bithiopheneacceptor or polycarbazole-acceptor copolymers. ${ }^{26}$ Conjugated polymers have found a wide range of applications in sensing, cellular imaging and optoelectronic devices ${ }^{27-29}$ (solar cells, light-emitting diodes, ${ }^{30}$ photovoltaic devices ${ }^{31}$ and organic thin film transistors). ${ }^{32,27}$

The interaction between conjugated polymers and phospholipids is a recently explored field which enhances the applications of the type of polymers. In biosensing, ${ }^{33}$ cationic conjugated polyelectrolytes supported on silica microspheres and overcoated with an anionic phospholipid provide "lipobeads" that serve as fluorescent sensors for phospholipase $\mathrm{A}_{2}$ activity. ${ }^{34}$ Similarly, the incubation of the conjugated polyelectrolyte-lipid complex with the enzyme phospholipase C causes the decrease of the emission intensity (turnoff sensor) and results in another lipase assay. ${ }^{35}$ The mechanism of the antimicrobial activity of a poly(phenylene ethynylene) based conjugated polyelectrolyte has been studied, and is based on the interaction with different phospholipids that are correlated with the ability of this polymer to selectively target bacteria cells over human cells. ${ }^{36-38}$

Phospholipids are also used to encapsulate semiconducting polymer nanospheres ${ }^{39}$ or hybrid nanoparticles (such as hydrophobic conjugated polymers and iron oxide $)^{40}$ as non-toxic fluorescent markers in cell imaging. Moreover ionophorephospholipid-polydiacetylene mixed vesicles are cation-selective color sensors at submillimolar concentrations which show significant ionic selectivity, in particular towards $\mathrm{Na}^{+}$and $\mathrm{K}^{+} \cdot{ }^{\mathbf{4 1}} \mathrm{A}$ cationic fluorene-phenylene polymer has also been used as a fluorescent membrane marker to visualize and monitor membrane processes. ${ }^{25}$

The intercalation of the neutral poly(9,9-dioctylfluorene), PFO, into the phospholipid bilayer of 1,2-dimyristoyl-sn-glycero3-phosphatidylcholine (DMPC) liposomes leads to fluorescent nanoparticles in solution in which the liposome acts as a carrier as well as a template controlling the polymer structure. ${ }^{3}$ PFO is an important blue-emitting polymer which shows a variety of secondary structures. ${ }^{42}$ In this polymer, random domains (socalled $\alpha$-phase) and more planar domains ( $\beta$-phase) frequently coexist although an efficient energy migration from the $\alpha$ to $\beta$ phase is produced. ${ }^{\mathbf{4 3 , 4 4}}$ The latter structure is characterized by an enhanced luminescence, a characteristic well-defined narrow absorption band at $438 \mathrm{~nm}$ and a well resolved fluorescence. ${ }^{\mathbf{4 3}}$ PFO $\beta$-phase is formed when the polymer is enclosed in natural $^{45}$ or synthetic phosphatidylcholines (PCs),,46 or in liposomes with other head groups. ${ }^{46}$ In general, the phospholipids with either longer alkyl chain lengths or less bulky and more hydrophobic head groups promote the formation of the $\beta$ phase more effectively. Polymer agglomeration within the liposome and the establishment of an improved interaction between the phospholipid alkyl chains seem to be the two driving forces which promote the phospholipid-polymer interaction, and induce the planarization of the PFO structure. ${ }^{\mathbf{4 6}}$ With natural lecithins, the reduction of the free volume in the inter-bilayer, the increase of the density of the bulk fatty acids and the orientation of double bonds of the phospholipid towards the bilayer centre, favour the formation of the PFO $\beta$ phase in soybean PC (S-PC) in which the linoleic acid chain (18 carbon atoms and two double bonds in the phospholipid alkyl chain, $18: 2$ ) is predominant, with respect to the egg PC (E-PC), where palmitic acid (16:0) and oleic acid (18:1) chains are dominant. $^{45}$

The dependence of the $\beta$-phase formation on the alkyl chain length of the substituent on the 9-carbon atom of fluorene, on the number of fluorene repeat units in the polymer backbone and on the defect concentration ${ }^{47}$ is well-known. The octyl chain has an optimal side chain length to form the $\beta$-phase, ${ }^{48}$ whilst it is more difficult to align and interdigitate longer side chains, even in solid films; this is considered as one of the driving forces in the planarization of the polyfluorene backbone. ${ }^{49}$ In addition, the $\beta$-phase is formed only when there are at least 9 repeat units in the polymer chain. ${ }^{50}$

In this work, we have combined molecular dynamics (MD) simulations with a variety of experimental techniques (infrared and electronic absorption spectroscopy, steady-state and timeresolved fluorescence emission, dynamic light scattering and differential scanning calorimetry) to study how oligo- and polyfluorenes are incorporated into DMPC liposomes in aqueous suspensions.

However, no single experimental technique can fully explain the observed behaviour, and since the overall goal is to understand the factors that control the formation of the $\beta$ phase, such as the interaction with the phospholipid bilayer, we have used molecular dynamics (MD) simulations to study the topography (in-membrane localization, orientation and conformation) of the compounds within the DMPC membranes. Because of the complexity of the system, we have carried out comparative MD simulations with model small molecules: benzene (B), biphenyl BP, and TF, Fig. 1. This provides information about how the structure of the compound affects its topography within the bilayer, and, therefore, helps us understand both the conformation and alignment oligomers adopt when enclosed in the liposome. Whilst extension of this to polymers is not without risk, the 


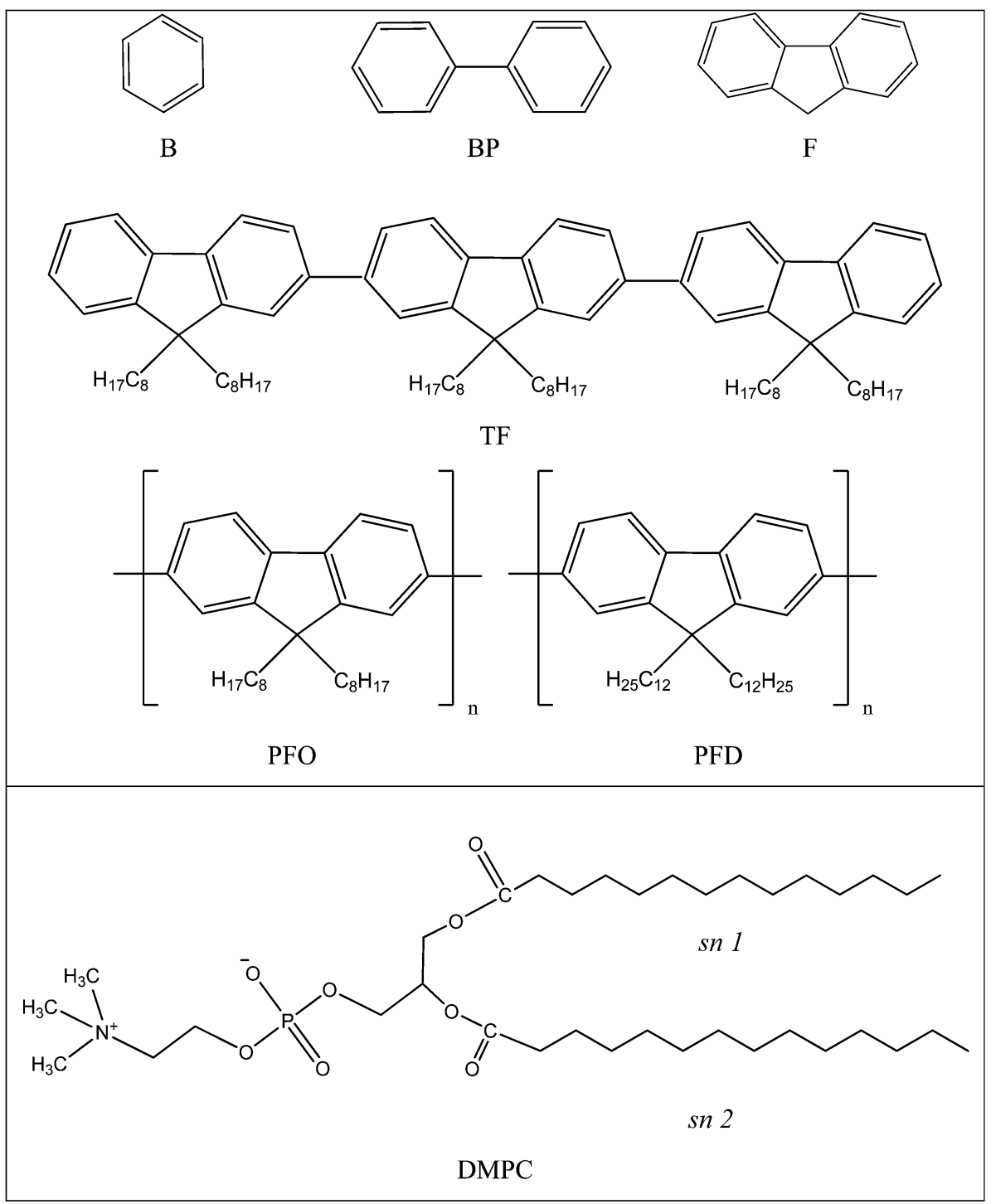

Fig. 1 Structures of benzene (B), biphenyl (BP), fluorene (F), tri-(9,9-di-n-octylfluorenyl-2,7-diyl) (TF), poly(9,9-di- $n$-octylfluorenyl-2,7-diyl) (PFO) poly(9,9-di-n-dodeylfluorenyl-2,7-diyl) (PFD) and 1,2-dimyristoyl-sn-glycero-3-phosphocholine (DMPC).

orientation of PFO within the phospholipid bilayer is very likely to be similar to that of the TF trimer, and can be related to the formation of the $\beta$-phase. In addition, the role of the fluorene alkyl side chain length and of the number of polymer repeat units in the formation of the $\beta$-phase when the polymer is incorporated into liposomes using tri-(9,9-di- $n$-octylfluorenyl-2,7-diyl) (TF), PFO and the poly(9,9-di-n-dodecylfluorenyl-2,7-diyl) (PFD) is studied. The validity of this approach is tested by comparison of the photophysics of these compounds with that of fluorene (F), in both DMPC and organic solvents. Although the sensitivity of the different techniques used and the computer time requirements for MD simulations have led to different molecular ratios of probe to phospholipid being used, we believe that this will not significantly affect the probe-phospholipid interactions, and the results do provide a reasonable picture of these systems.

\section{Experimental}

\section{Materials and sample preparation}

Phospholipid 1,2-dimyristoyl-sn-glycero-3-phosphatidylcholine was purchased from Sigma, fluorene from Fluka, tri-(9,9-di- $n$ octylfluorenyl-2,7-diyl) from American Dye Source, and polyfluorene poly(9,9-di- $n$-octylfluorenyl-2,7-diyl $)\left(M_{\mathrm{w}}=58.2\right.$ $\left.\mathrm{kg} \mathrm{mol}^{-1}\right)$ and poly$(9,9-$ di- $n$-dodecylfluorenyl-2,7-diyl $)\left(M_{\mathrm{w}}=9.4\right.$ $\mathrm{kg} \mathrm{mol}^{-1}$ ) were purchased from Aldrich and used without further treatment, Fig. 1. Chloroform was purchased from Sigma-Aldrich and cyclohexane and ethanol were purchased from Merck. All of them were of spectroscopic grade. Molar concentrations for the oligomer and polymers are calculated in terms of the repeat units.

Trizma-HCl ( $\mathrm{pH}=7.4)$ buffer solution was prepared with Trizma base (Sigma) and $\mathrm{HCl}\left(37 \%\right.$ and $d=1.19 \mathrm{~g} \mathrm{~cm}^{-3}$, Panreac). 
The alkyl chain deuterated PFO $\left(\mathrm{PFO}_{34}\right)$ for IR experiments was kindly provided by Professor U. Scherf (Bergische Universität Wuppertal, Germany) and Dr A. T. Marques. The synthesis and characterization have been described elsewhere. ${ }^{\mathbf{1 1}}$

Liposomes were prepared by the thin film hydration method. DMPC and DMPC-PFO mixtures in chloroform were evaporated to dryness by bubbling argon. The resulting films were hydrated with Trizma-HCl buffer $(\mathrm{pH}=7.4)$ to give a final phospholipid concentration around $9 \times 10^{-5} \mathrm{M}$ and $\mathrm{PFO}$ concentrations varying from $1 \times 10^{-6}$ to $1 \times 10^{-5} \mathrm{M}$ (except for DSC experiments, which require a higher phospholipid concentration, around $4 \times 10^{-2} \mathrm{M}$, with $\mathrm{PFO}$ concentrations varying from $1 \times 10^{-3}$ to $4 \times 10^{-2} \mathrm{M}$ ).

To form the liposomes, the samples were kept for 10 minutes at a temperature $\left(40{ }^{\circ} \mathrm{C}\right)$, well-above the DMPC transition temperature $\left(23.5^{\circ} \mathrm{C}\right)$, and then were vortexed three times for 1 minute. Subsequently, they were alternately sonicated and heated at $40^{\circ} \mathrm{C}$ to the same temperature, for $5 \mathrm{~min}$ each, during $1 \mathrm{~h}$. The samples were then kept at this temperature while stirring for another hour. Under such conditions, the obtained liposomes are mainly unilamellar, as confirmed by transmission electron microscopy (TEM). ${ }^{52}$

\section{Apparatus and methods}

Absorption spectra were recorded on a Shimadzu 2501 PC UV visible spectrophotometer. For steady - state luminescence measurements, a Shimadzu RF-5301 PC spectrofluorimeter was used. The excitation wavelength was $387 \mathrm{~nm}$, and excitation and emission slits were 3 and $1.5 \mathrm{~nm}$, respectively. The spectra were recorded at $25.00^{\circ} \mathrm{C} \pm 0.01{ }^{\circ} \mathrm{C}$, above the gel-liquid crystalline phase transition temperature of DMPC, which corresponded to the condition that showed the least background scattering.

Fluorescence quantum yields were calculated using as standards: tryptophan in aqueous solution (quantum yield 0.15 for a concentration $10^{-4} \mathrm{M}$ at $\mathrm{pH} 6.1$, excitation wavelength 270 $\mathrm{nm})^{53}$ for $\mathrm{F}$ and quinine bisulfate $\left(0.546\right.$ in $1 \mathrm{~N} \mathrm{H}_{2} \mathrm{SO}_{4}$, excitation wavelength around $350 \mathrm{~nm})^{54}$ for TF, PFO and PFD according to the procedure described by Eaton. ${ }^{54}$

Time-resolved fluorescence measurements were carried out using the single photon counting technique with picosecond time resolution on a home-built apparatus that has been described elsewhere. ${ }^{55}$ Excitation was at $392 \mathrm{~nm}$ and the emission was recorded at 440, 465 and $500 \mathrm{~nm}$. Fluorescence decays and the instrumental response function (IRF) were collected using 4096 channels (with 0.814 ps per ch), until $5 \times 10^{3}$ total detected counts at maximum were reached. De-convolution of the fluorescence decay curves was performed using the method of the modulation functions previously implemented by Striker. ${ }^{56}$

Differential scanning calorimetry (DSC) measurements were performed on a MDSC Q-200 TA differential scanning calorimeter equipped with a refrigerated cooling system (RCS). A $50 \mathrm{~mL}$ $\min ^{-1}$ nitrogen purging gas flow was used. The samples were initially cooled to $-2{ }^{\circ} \mathrm{C}$ and pre-equilibrated at this temperature for 30 minutes. Three heating-cooling cycles between $-2{ }^{\circ} \mathrm{C}$ and $80^{\circ} \mathrm{C}$ were carried out for each sample with a heating rate of $1.5{ }^{\circ} \mathrm{C} \min ^{-1}$. Very good reproducibility was obtained between consecutive scans.

Dynamic light scattering (DLS) measurements were carried out using a Zetasizer Nano ZS90 (Malvern, UK) to determine the hydrodynamic diameter and the polydispersity index (PI) of the liposomes. ${ }^{57}$ The DLS technique measures the Brownian motion of the particles and correlates it with the hydrodynamic radius, $r_{\mathrm{h}}$, of a particle defined by the Stokes-Einstein equation.

$$
r_{\mathrm{h}}=\frac{k T}{3 \pi \eta D}
$$

where $D$ is the translational diffusion coefficient $\left(\mathrm{m}^{2} \mathrm{~s}^{-1}\right), k$ is Boltzmann's constant, $T$ is the absolute temperature (K), and $\eta$ is the coefficient of viscosity ( $\mathrm{Pa} \mathrm{s}$ ). A $632 \mathrm{~nm} \mathrm{He} / \mathrm{Ne}$ laser beam crosses the sample and the scattered light at $90^{\circ}$ is analysed considering the size distribution by intensity and by volume of scattered light. The DLS measurements were performed at $25{ }^{\circ} \mathrm{C}$.

Infrared measurements were performed on a Bomem (MB104) Fourier transform spectrometer equipped with a deuterated triglycine sulphide (DTGS) detector and $\mathrm{Zn} / \mathrm{Se}$ optics. Data collection was performed with $2 \mathrm{~cm}^{-1}$ spectral resolution and 128 accumulations.

\section{Simulation details}

System. The effect of a number of different aromatic molecules, containing one, two and three rings, in a fully hydrated DMPC bilayer was studied by molecular dynamics (MD) simulation. The topology for each aromatic residue (benzene, biphenyl, fluorene, and a 3-ring polyfluorene) was generated by the PRODRG server, ${ }^{58}$ while DMPC and Simple Point Charge (SPC) water were described using the original definitions of the GROMOS 53a6 force field. ${ }^{59}$ A DMPC bilayer was used, consisting of 128 phospholipid molecules equally distributed by two leaflets and 3655 SPC water molecules, as made available by Kukol. ${ }^{60}$ It should be noted that, for simplicity, a single molecule of solute was embedded in the DMPC bilayer. Such an approach is suitable for studying the interaction of each solute with the surrounding lipids, as well as assessing the respective conformation and preferential positioning relative to the bilayer center.

In order to circumvent timescale limitations associated with a full description of the process of insertion, molecules were directly incorporated into a pre-equilibrated and fully hydrated DMPC bilayer. Different positioning alternatives were tested for the insertion of the relevant molecules into the bilayer, rapidly converging to the same overall positioning in each case.

Parameters and data analysis. All MD simulations were carried out in the NpT ensemble and under periodic boundary conditions, resorting to the GROMACS package, version 4.5.4 ${ }^{61}$ and the GROMOS $53 \mathrm{a} 6$ force field. ${ }^{59}$ A standard time step of 2 fs was used for both the equilibration and production runs. Nonbonded interactions were computed on the basis of a neighbour list, updated every 10 steps. Long-range electrostatics was computed using the particle mesh Ewald (PME) method, as recommended for charged polymer simulations. For 
Lennard-Jones energies, a cut-off of $1.4 \mathrm{~nm}$ was applied. Temperature and pressure were coupled to the Berendsen external baths maintained at $314 \mathrm{~K}$ and 1 bar, with coupling constants of 0.1 and $0.5 \mathrm{ps}$, respectively. To obtain a starting configuration, each system was firstly subjected to an energy minimization step. The systems were then left to evolve up to 120 ns, using the LINCS algorithm ${ }^{62}$ to keep bonds containing $\mathrm{H}$ atoms under positional restraint conditions. The first $40 \mathrm{~ns}$ were considered sufficient to attain equilibrated systems, while the last $80 \mathrm{~ns}$ of production runs were subsequently subjected to standard analysis, such as atom-atom (group-group) distance distributions and radial distribution functions (rdf). MD trajectories were visualized, and configuration images extracted using the VMD 1.8.6 software. ${ }^{63}$ It should be noted that for such a system, considerations based on statistical inefficiency assessment indicate that it takes about 100 ps to renew the overall information of the system, which makes the total simulation length more than sufficient for an accurate description of the system. ${ }^{64}$

\section{Results and discussion}

Molecular dynamic simulations give fundamental information on the location, diffusion and interaction of F, TF, PFO and PFD within the phospholipid bilayer. The interaction of the probes with the phospholipids in the liposome has been further studied with IR and DSC. The dynamic light scattering results allow estimation of the effect of these compounds on the size of liposomes. Moreover, the spectroscopic characteristics (absorption, steady-state and time-resolved fluorescence) of the probes incorporated into DMPC liposomes are compared with the spectroscopic characteristics of the same probes in typical organic solvents and the results are related to those of the other experimental techniques and molecular dynamic simulations.

\section{Molecular dynamics simulations}

MD simulations were carried out with a single molecule of $\mathrm{B}$, $\mathrm{BP}, \mathrm{F}$ the trimer (TF) was enclosed in a fully hydrated DMPC bilayer (Fig. 1), consisting of 128 phospholipid molecules equally distributed by two leaflets to gain insight into the topology of these molecules incorporated into the membrane and of the interaction between these molecules and the phospholipids.

In Fig. 2B, the normalized probability density profiles are shown for the centres of mass of the molecules inserted into the DMPC bilayer relative to the $Z$-axis (where the $Z$-coordinate represents the normal to the bilayer plane) with the origin in the center of the bilayer, and compared with the normalized density profiles of the DMPC heads, tails and the water that surrounds
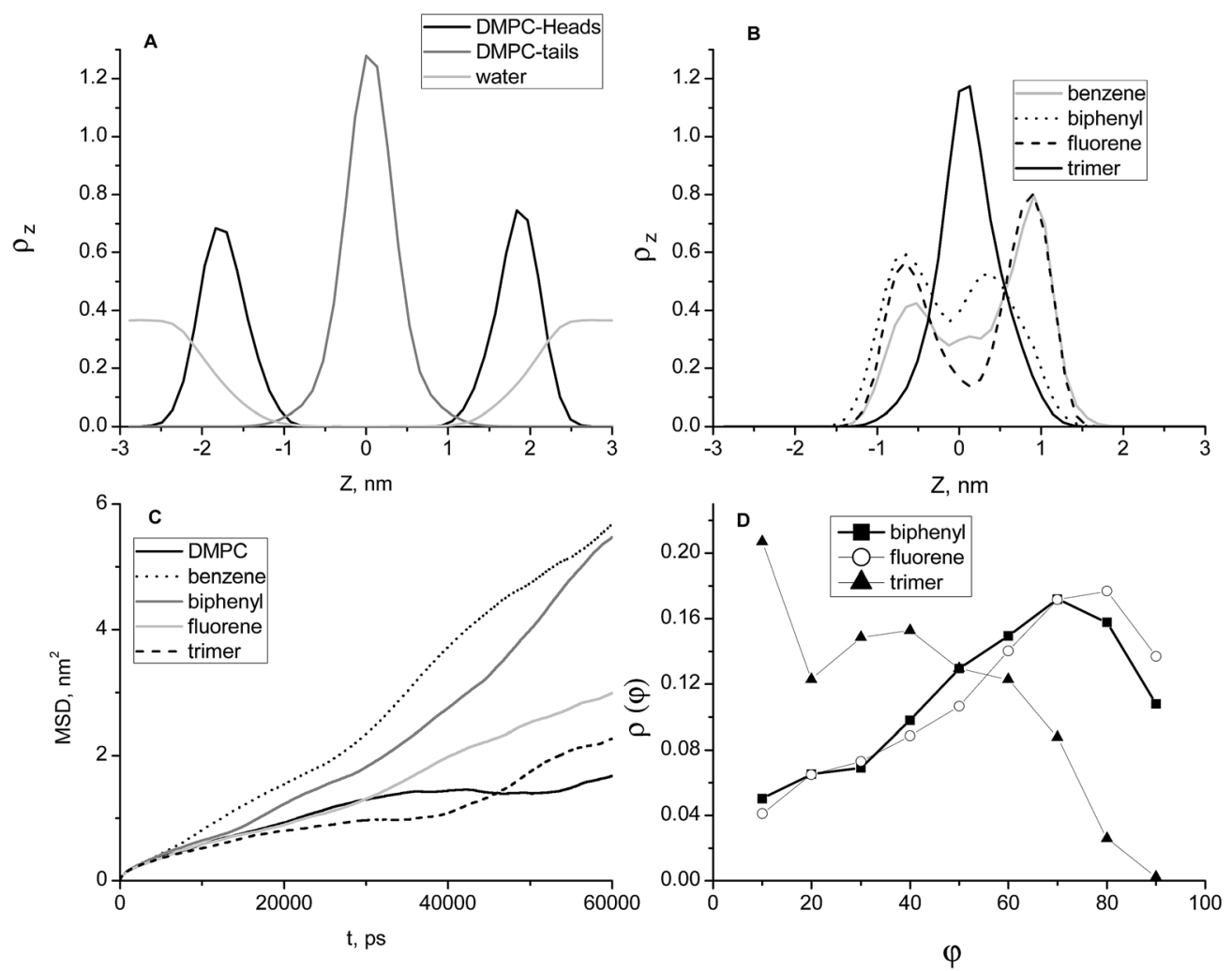

Fig. 2 (A) Probability density profiles for the DMPC heads (solid black line), tails (solid gray line) and water (solid pale gray line) relative to the $Z$-axis. Note that the $Z$-coordinate represents the normal to the bilayer plane ( $Z=0$ in the centre of the bilayer) and that each distribution was normalized to unity. (B) Probability density profiles for the centres of mass of the indicated molecules inserted in the DMPC bilayer. (C) Mean square displacement (MSD) of the molecules under study within the DMPC bilayer. (D) Probability distribution for the angle between the main axes of the indicated molecules, and the interface plane of the DMPC bilayer (the lines are meant to be a guide to the eye). Fig. 3 indicates the molecular axes in the situations that are represented. 
the bilayer (Fig. 2A). It is shown that the molecules under study are embedded in the hydrophobic region of the bilayer, with the benzene, biphenyl and fluorene displaying a preferential positioning roughly midway between the center of the bilayer $(Z=0)$ and the lipid/water interface. The two smaller molecules (benzene and biphenyl) are those that more frequently switch between leaflets, as is confirmed by the solute diffusion estimated from the mean square displacement profiles (MSD) shown in Fig. 2C. In contrast, the trimer tends to reside in the center of the bilayer (Fig. 2B) and is the molecule that diffuses more slowly (Fig. 2C). Fluorene diffusion shows an intermediate behavior between those of the smaller molecules (B and BP) and that of the trimer, as expected from its intermediate molecular size. It is seen that all the molecules span a considerable fraction of the bilayer thickness, covering both leaflets. As such, and irrespective of some preferential positioning, there is no indication of either of these molecules, including the trimer, being trapped or unable to move.

In terms of orientation, it is seen that the main axes of biphenyl and fluorene molecules are parallel to the lipid chains, while the trimer backbone remains mostly perpendicular to the latter as is shown by the probability distribution of the angle formed between the main axes of the molecules and the interface plane of the DMPC bilayer (Fig. 2D) and also in the snapshots (Fig. 3). Moreover, the alkyl side chains on the 9-position of the trimer have some propensity to extend towards the interface, being interdigitated with the alkyl chains of the phospholipid, as illustrated in Fig. 3.

These results are comparable with those found in the literature for neutral aromatic compounds such as pyrene in the

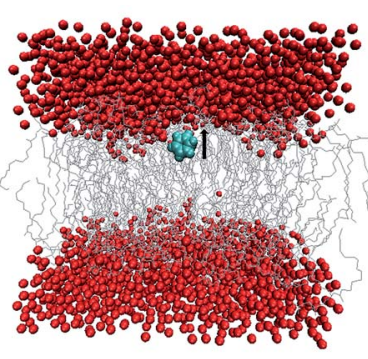

DMPC/benzene

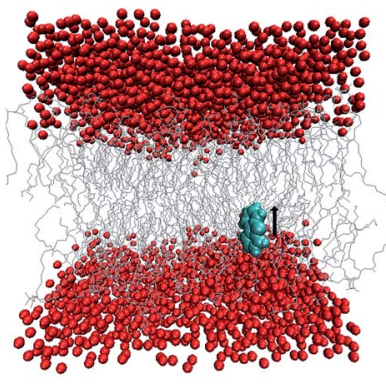

DMPC/fluorene

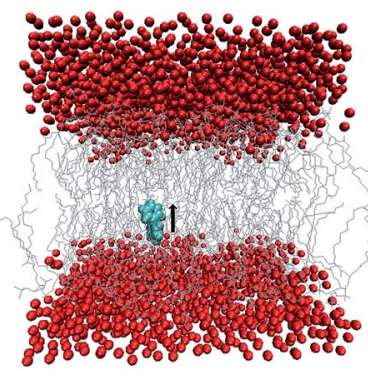

DMPC/biphenyl

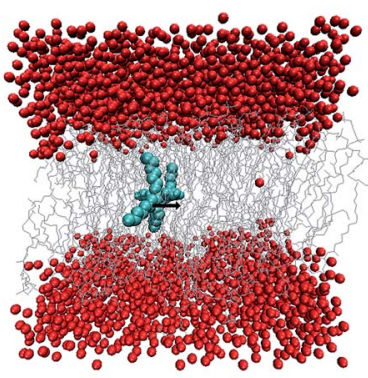

DMPC/polyfluorene
Fig. 3 Snapshots extracted from the MD simulations illustrating typical positioning and conformation of the residues embedded in the DMPC bilayer. The arrows represent schematically the molecular axes in each case.
DPPC bilayer. ${ }^{65}$ Pyrene is mainly located in the hydrophobic acyl chain region close to the glycerol groups of lipid molecules. Its orientation is dependent on the phase of the membrane. In the fluid phase (conditions of our study) pyrene stands upright in parallel to the phospholipid alkyl chains (as we have concluded with $\mathrm{B}, \mathrm{BP}$ and $\mathrm{F}$ ) while in the gel phase, the orientation is affected by the tilt of lipid acyl chains. ${ }^{65}$ Pyrene incorporated into the 1-palmitoyl-2-oleoyl-sn-glycero-3-phophocholine (POPC) bilayer also prefers to be located in the hydrophobic acyl chain region close to the glycerol group of the lipid bilayer. ${ }^{66}$

There are numerous other examples of aromatic molecules, even with heteroatoms, that tend to be in the hydrophobic part or the phospholipid bilayers orientated perpendicular to the membrane interphase. 6-Propionyl-2-( $N, N$-dimethylamino) naphthalene (PRODAN) is in the bilayer with the dimethylamino group pointing toward the membrane's center and the carbonyl oxygen toward the membrane's interface. ${ }^{67}$ With pyrroloquinolines in DPPC, it has been found that the hydrophobic aromatic parts of the probes are located inside a more ordered hydrophobic lipid chain region of DPPC. The depth of the probe localization is gradually shifted deeper inside the hydrocarbon core of the bilayer with the increase of the number of aromatic rings. ${ }^{68}$ Kyrychenko and Waluk found that the probe-lipid hydrogen-bonding contributes weakly to the favorable location of the azaaromatic probes inside the DPPC bilayer, so that the probe location is mainly driven by electrostatic dipole-dipole and van der Waals interactions. ${ }^{68}$

Similarly, MD simulations carried out with neutral and charged lidocaine in the DMPC bilayer above the transition temperature show that the uncharged form is located down in the upper part of the lipid tails presumably perpendicular to the normal orientation while the charged molecules exhibit a defined position at the lipid head group region with preferential orientation along the bilayer normal. ${ }^{69}$ Considering the diffusion behaviour, the MD simulations indicate that charged lidocaine has a rather restricted motion whereas the uncharged molecules, as we have observed in our systems, are free to diffuse in the lateral direction as well as to jump from one side of the bilayer to the other. ${ }^{69}$

Order analysis has been carried out to investigate the influence of the spectroscopic probes on the bilayer structure through the alkyl chain order parameter $S_{\mathrm{CD}}$ (Fig. 4), which characterizes the average conformational ordering of lipid hydrocarbon chains, and is frequently obtained using the quadrupolar splitting in deuterium NMR spectra. The results gathered in Fig. 4A show that both the fluorene molecule and biphenyl induce an increased organization in the hydrophobic part, towards the interface. For the fluorene molecule, this effect extends to the interior of the bilayer, probably due to its rigid character.

Although only one molecule of solute inserted in a 128 lipid bilayer represents a small effect over the bilayer properties, the increase in the order found for the DMPC/fluorene system was responsible for a visible decrease in the area per lipid and a consequent increase in the bilayer thickness (see Fig. S1 in the $\mathrm{ESI} \dagger)$. 


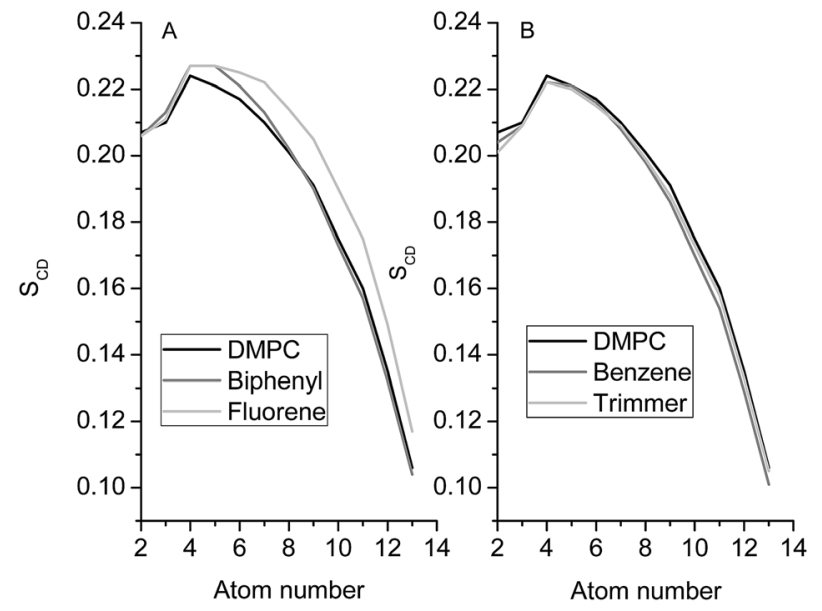

Fig. 4 Alkyl chain order parameter, $S_{C D}$, estimated along the bilayer depth for DMPC chains (and averaged over the sn-1 and sn-2 chains) in the presence of the indicated solutes.

$S_{\mathrm{CD}}$ also shows that the acyl chains of the DPPCs next to pyrene molecules are considerably more ordered than the lipids on average in the fluid phase ${ }^{65}$ The same effect is observed on the POPC acyl chain order parameters upon incorporation of pyrene with changes which are rather small in global terms, but with significant local changes; again pyrene induces ordering of nearby phospholipid chains in disordered phases and conversely for ordered bilayers. ${ }^{66}$ However, in the presence of cholesterol and in the fluid phase, pyrene increases the order of the bilayer. ${ }^{66} \mathrm{~A}$ decrease of the ordering of those hydrocarbon chains that are closest to the pyrene in the gel phase has also been observed with DPPC. ${ }^{65}$

The less significant effect of B and BP compared to F on $S_{\mathrm{CD}}$ is probably a consequence of them being smaller and less rigid molecules than F. For the trimer, the fact that the majority of the molecules are in the center of the bilayer and that only the flexible octyl chains are orientated towards the phospholipid alkyl changes could justify the small effect observed on the alkyl chain order parameter.

\section{Infrared study}

To gain insight into the interaction between DMPC and PFO, the IR spectra of the films of neat DMPC, the alkyl-chain deuterated PFO- $\mathrm{d}_{34}$, and DMPC-PFO- $\mathrm{d}_{34}$ mixture with a molar ratio $1: 1$ were prepared by drop-casting chloroform solutions of the compounds onto $\mathrm{CaF}_{2}$ windows.

Fig. 5 shows the $1500-1480 \mathrm{~cm}^{-1}$ spectral regions of the IR spectra of neat DMPC and PFO- $\mathrm{d}_{34}$, and DMPC-PFO- $\mathrm{d}_{34}$ mixture. PFO- $\mathrm{d}_{34}$ gives rise to a single band at $1457 \mathrm{~cm}^{-1}$. On the other hand, both neat DMPC and DMPC-PFO- $\mathrm{d}_{34}$ mixture, besides the band at $1457 \mathrm{~cm}^{-1}$, also give rise to a band at higher wavenumbers, $1467 \mathrm{~cm}^{-1}$.

Wang et al. ${ }^{70}$ showed that 2-alkyl-7,7,8,8-tetracyanoquinodimethane (which belongs to the $\mathrm{C}_{n}$-TCNQ family of compounds, where $n$ is the length of the alkyl chain), exhibits a stacked structure in a head-to-head manner across the center of

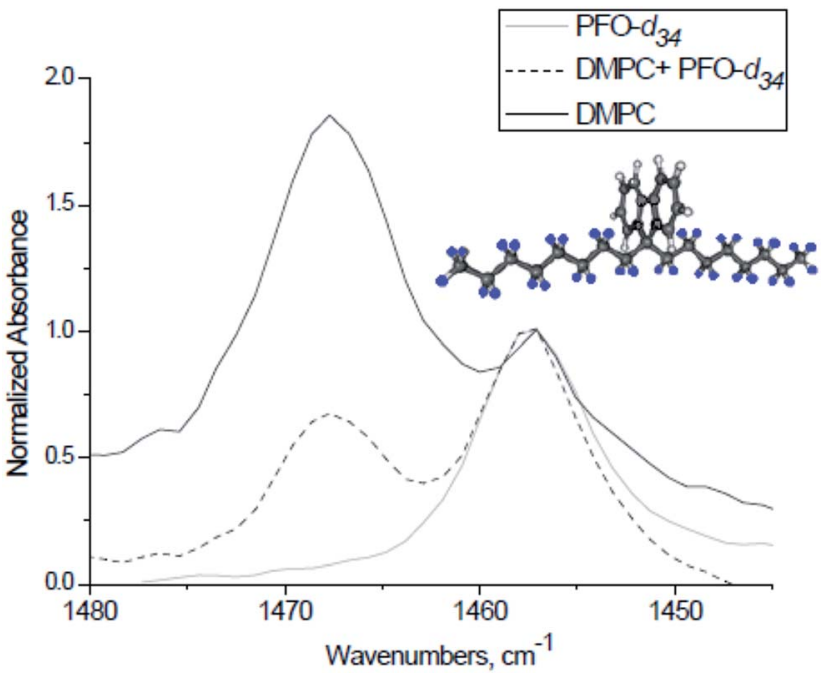

Fig. $51500-1480 \mathrm{~cm}^{-1}$ spectral region of the IR normalized absorption spectra of cast films of DMPC (from $20 \mu \mathrm{L}$ of DMPC $5 \times$ $10^{-3} \mathrm{M}$ in chloroform, black solid line), PFO- $d_{34}$ (from $40 \mu \mathrm{L}$ of PFO$\mathrm{d}_{34} 2.5 \times 10^{-3} \mathrm{M}$ in chloroform, light gray solid line) and DMPC-PFO$\mathrm{d}_{34}$ (from $20 \mu \mathrm{L}$ of DMPC $5 \times 10^{-3} \mathrm{M}$ and $40 \mu \mathrm{L}$ of PFO- $\mathrm{d}_{34} 2.5 \times 10^{-3}$ $M$ in chloroform, black dashed line). Inset: structure of the alkyl-chain deuterated $\mathrm{PFO}-\mathrm{d}_{34}$ with deuterium atoms in blue.

symmetry to form a double layer of -TCNQ groups. The trans$z i g$-zag planes of the alkyl chains are parallel to each other, protrude oppositely from the TCNQ layers and are interdigitated with each other (with the majority of the $-\mathrm{CH}_{2}$ groups in the interdigitated part). For this family of compounds, the bands around 1470 and $1460 \mathrm{~cm}^{-1}$ were assigned to the noninterdigitated and interdigitated part of the alkyl chains, respectively. Based on the work of Wang et al. ${ }^{70}$ the bands that appear in the IR spectrum of DMPC, as well as those of the DMPC-PFO-d $\mathrm{d}_{34}$ mixture, with maxima at 1467 and $1457 \mathrm{~cm}^{-1}$, are assigned to the non-interdigitated and interdigitated parts of the DMPC alkyl chains, respectively.

In the IR spectrum of the DMPC-PFO- $\mathrm{d}_{34}$ mixture, there is a significant decrease in the intensity of the band at $1467 \mathrm{~cm}^{-1}$, compared with the respective band in the IR spectrum of neat DMPC, assigned to the non-interdigitated part of the DMPC alkyl chains. It should be mentioned that at this wavenumber PFO- $\mathrm{d}_{34}$ does not exhibit any band. This observation is compatible with the interdigitation between the octyl chain of the polymer and the alkyl chain of the phospholipid into the bilayer, as indicated by molecular dynamics simulations (Fig. 3). The topology shown in the snapshots of the DMPC/ polyfluorene system is in good agreement with the increase of the intensity of the interdigitated $-\mathrm{CH}_{2}$ alkyl DMPC group band $\left(1457 \mathrm{~cm}^{-1}\right)$ shown by the IR results (Fig. 5 ).

\section{Differential scanning calorimetry}

The interaction between the fluorescent probes (F, TF, PFO and PFD) and DMPC has been further studied using DSC through the changes produced in the main phase transition temperature of DMPC upon the incorporation of the probes. In this, the 
lipids undergo a transition from the ordered gel-state to a disordered fluid-state which can be affected by the molecules dissolved into the lipid bilayer. The transition temperature variations $(\Delta T)$, presented in Table 1 for three different DMPC to probe ratios, are calculated as the difference between the main transition temperature of liposomes in the presence $\left(T_{\mathrm{m}}\right)$ and absence $\left(T_{\mathrm{m}}^{0}\right)$ of the fluorene derivatives.

In addition, the incorporation of the $\mathrm{F}, \mathrm{TF}, \mathrm{PFO}$ and PFD into the phospholipid bilayer leads to a decrease of the height and to an increase of the width of the peak, as is shown in the thermograms (Fig. S2 of the ESI $\dagger$ ), confirming experimentally that all the compounds are incorporated into the phospholipid bilayer and affect the main DMPC-liposome phase transition, in agreement with the MD simulations (Fig. 3). The increase of the peak width (Fig. S2 $\dagger$ ) indicates a decrease in the cooperativity among the acyl chains of the phospholipids for the transitions following the probe insertion into the lipid bilayer. ${ }^{2}$

The shift of the transition varies depending on the structure of the probe involved, indicating differences in the interaction with the phospholipid (Table 1). With F, a clear negative shift of $T_{\mathrm{m}}$ is seen, similar to those found in the literature for this compound enclosed in DMPC liposomes. ${ }^{2}$ Negative shifts are also described with 2-nitrofluorene and 3-nitrofluoranthene. ${ }^{71}$ However, with the two polymers studied: PFO and PFD, positive shifts of $T_{\mathrm{m}}$ are observed (Table 1, following the order PFD > PFO) indicating the formation of more ordered structures. ${ }^{72}$

Shifts of the main transition temperature of dioctadecyldimethylammonium bromide liposomes (DODAB, $\left.45.34{ }^{\circ} \mathrm{C}\right)$ to higher temperatures $\left(45.24^{\circ} \mathrm{C}\right)$ have been observed upon incorporation of the 18-2-18 cationic gemini surfactant into DODAB before the vesicles are formed. These gemini surfactants have identical tetraalkylammonium head groups connected by a saturated alkyl chain with two carbon (s) atoms and two symmetric saturated alkyl tails with 18 carbon atoms (m) and they are generally represented by $\mathrm{m}-\mathrm{s}-\mathrm{m}$.

However, gemini surfactants with shorter lateral alkyl chain lengths promote shifts of the main transition to lower temperatures (12-2-12 and 16-2-16 to 31.38 and $38.82{ }^{\circ} \mathrm{C}$, respectively). ${ }^{72}$ In the case of DODAB and 18-2-18, the surfactants have the same alkyl chain tail length, and MD simulations indicate that the terminal methyl groups of 18-2-18 reach the central part of the bilayer, increasing the lateral packing, which is compatible with the main transition being displaced to a higher

Table 1 Transition temperature variation $(\Delta T)$ with mean standard deviation $( \pm S D)$ of DMPC liposomes with various concentrations of fluorene based probes enclosed. $\Delta T=T_{m}-T_{m}^{0}$, where $T_{m}^{0}$ is the transition peak temperature of pure DMPC liposomes and $T_{m}$ that in the presence of the probe

\begin{tabular}{llrll}
$\begin{array}{l}\text { DMPC }: \text { probe } \\
\text { ratio }\end{array}$ & F & TF & PFO & \multicolumn{1}{l}{ PFD } \\
\hline $10: 1$ & $-4.02 \pm 0.30^{a}$ & $-0.22 \pm 0.36$ & $0.53 \pm 0.20$ & $1.41 \pm 0.20$ \\
$2: 1$ & $-3.05 \pm 0.33$ & $-0.78 \pm 0.30$ & $0.51 \pm 0.23$ & $0.66 \pm 0.30$ \\
$1: 1$ & $-3.10 \pm 0.33$ & $0.16 \pm 0.40$ & $0.51 \pm 0.25$ & $1.25 \pm 0.20$ \\
& & & \\
${ }^{a}$ Ratio $11: 1$ in the case of PFO. & & &
\end{tabular}

temperature. ${ }^{72}$ With the other two gemini surfactants (12-2-12 and 16-2-16), MD simulations indicate the formation of lower density regions in the core of the bilayer due to the small tails of the gemini surfactants compared with DODAB, and the presence of high charge density regions on the surface due to the two charged heads of gemini separated by a small spacer. Both factors justify the opposing behaviour observed in the transition temperature of DODAB depending on the structure of the m-s-m surfactant. ${ }^{72}$ Similar effects have been observed upon addition of the same surfactants (12-2-12 and 18-2-18) to dipalmitoylphosphatidylcholine : cholesterol (DPPC : Chol) liposomes. ${ }^{73}$ The main transition temperature of DPPC : Chol is shifted from 40.0 to $28.5^{\circ} \mathrm{C}$ by $12-2-12$ and from 41.0 to $46^{\circ} \mathrm{C}$ by 18-2-18. MD simulations again indicate that 12-2-12 embedded in the membrane provokes a strong reduction of density close to the bilayer interleaflet, while no evidence of disruption is seen with 18-2-18. Moreover, the latter adopts a conformation and a relative vertical positioning similar to the phospholipids, and a slight interdigitation between the surfactant and phospholipid chains is observed by MD, which explains the increase of order suggested by DSC. ${ }^{73}$ This is in agreement with the MD simulations and IR studies carried out for TF. The snapshots in Fig. 3 show that TF is placed into the core of the DMPC bilayer in the interleaflet region with the octyl-chain of the polymer orientated towards the alkyl chain of the phospholipid and partially interdigitated with them. This is in agreement with the IR results, and provides a ready explanation for the positive change in the transition temperature of DMPC liposomes.,46 Based on this discussion, it is plausible that the slightly larger shift of the main transition temperature induced by PFD than that induced by PFO is because the alkyl chain length of PFD is closer to that of DMPC, inducing a higher order into the bilayer core. It is also reasonable that $\mathrm{TF}$, with a similar location to that of the polymers within the membrane (as shown by MD simulations, Fig. 3) but with fewer repeat units in the backbone, shows intermediate thermotropic behaviour on the phase transition between that of $\mathrm{F}$ and of the polymers (Table 1). It is not expected to induce as much order into the membrane as the polymers, due to the smaller number of repeat units.

A peak broadening and a significant increase of $T_{\mathrm{m}}$ have been also observed upon addition of very low concentrations of Pluronic L-61 $\left(\mathrm{PEO}_{2}-\mathrm{PPO}_{30}-\mathrm{PEO}_{2}, 0.02 \mathrm{wt} \%\right)$ to DPPC. In the model proposed, L-61 is aligned with the phospholipids of the two leaflets of the bilayer. ${ }^{74}$

However, when the cationic polyfluorene $[9,9-\operatorname{bis}(6-N, N, N$ trimethylammonium)hexyl]fluorene-phenylene (HTMA-PFP) is added to preformed vesicles of 1,2-dimyristoyl-sn-glycero-3phospho-rac-(1-glycerol) sodium salt (DMPG) no changes are observed in the height, width or $T_{\mathrm{m}}$ in the thermograms, which indicates that the cooperativity of the transition is preserved and the polymer does not disrupt the overall structure of the lipid bilayer. ${ }^{25}$ With the neutral PFO and PFD added in our systems before vesicle formation with DMPC phospholipids, the polymers can become incorporated into the inner part of the bilayer, leading to the effect on the lipid bilayer structure and, in consequence, on the transition temperature. 
Considering both the thermal behaviour discussed above with different amphiphiles (DODAB and DPPC) and the gemini surfactants (12-2-12, 16-2-16 and 18-2-18), and our MD and IR results, we believe that the increase in the $T_{\mathrm{m}}$ induced by PFO and PFD is due to the fact they are located in the centre of the bilayer, with their poly(fluorene) backbones orthogonal to the alkyl chains of the phospholipid and the PF alkyl chains interdigitated with the end part of the alkyl chains of the phospholipid, thus increasing bilayer ordering and transition temperature. In contrast, fluorene is distributed across the membrane, with a tendency to be close to the headgroup region (Fig. 2 and 3) which may provoke a reduction of density close to the bilayer interleaflet (Fig. 4) without increasing ordering of the alkyl chains of the phospholipid. This leads to the expected decrease in the transition temperature.

\section{Dynamic light scattering}

Fluorene and PFO were incorporated into phospholipid bilayers before vesicle formation, and their effect on the size of DMPC vesicles was studied through dynamic light scattering. The main results are summed up in Fig. 6. In both cases, monomodal size distribution curves were obtained. These were broader for PFO, and the average particle radius for vesicles with $\mathrm{F}$ was $77 \pm 10 \mathrm{~nm}$ and for vesicles with PFO was $131 \pm 31$ $\mathrm{nm}$. From the standard deviation, it can be clearly seen that the samples with PFO show a greater polydispersity than those with F indicating that the nature of the probe affects both the size of the vesicles obtained and their distribution. This is corroborated by the variation of the liposome size upon addition of various concentrations of $\mathrm{F}$ and PFO as shown in Fig. 6A. Data have been normalized dividing the radius by that of the free liposomes. In both cases a decrease of liposome size is observed upon increasing the probe concentration. However, the decrease is more significant for PFO (slope $-67732 \pm 8752$ ) than for $\mathrm{F}$ (slope $-43537 \pm 3377$ ) showing a larger effect of the polymer on the vesicle size.
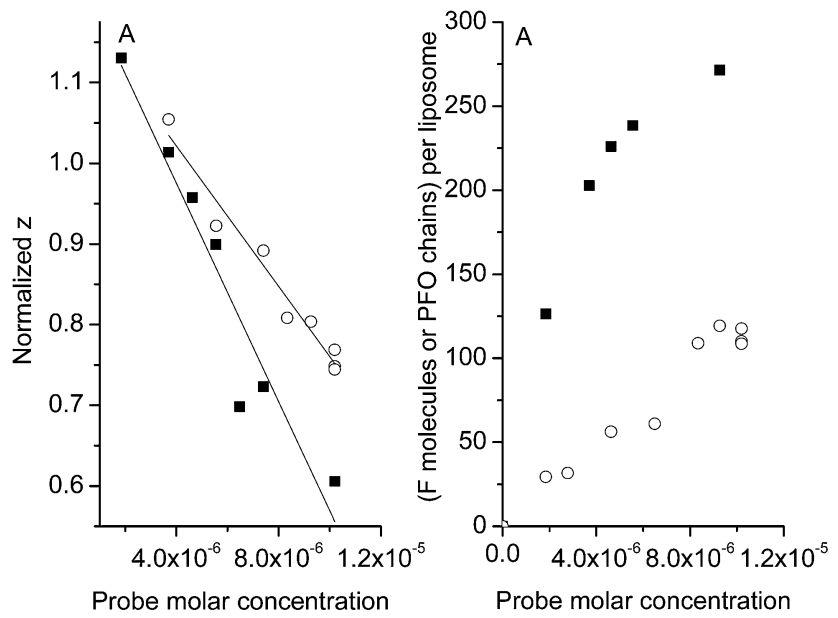

Fig. 6 (A) Changes of normalized DMPC liposome radius as a function of $F$ (empty black circles) and PFO (black squares) concentration. (B) Number of $F$ molecules or PFO chains per liposome (DMPC). In both cases, the DMPC concentration was $9.38 \times 10^{-5} \mathrm{M}$.
A decrease of vesicle size upon addition of the neutral triblock copolymer Pluronic F-127 to L- $\alpha$ phosphatidylcholine (egg PC, E-PC) and DPPC in a concentration range between 0.1 and $0.5 \%$ wt was described and attributed to a solubilisation process with most of the large unilamellar vesicles destroyed, yielding many bilayers disks of much smaller sizes in coexistence with small, but moderately enlarged F-127 modified unilamellar vesicles. ${ }^{74}$ Johnsson et al. also observed that the Pluronic P105 and P85 added to preformed vesicles tend to promote a reduction of EPC liposome size upon heating the samples to $55^{\circ} \mathrm{C}$ in an irreversible process, regardless of whether the samples were prepared by sonication or extrusion. ${ }^{75}$ However, as Cryo-TEM also showed smaller liposomes instead of bilayers disks, they concluded that the higher curvature needed to decrease the polymer repulsion at a high polymer surface concentration was achieved by liposomal size reduction. This was only observed at a temperature for which the interaction between the polymer and the phospholipid is achieved..$^{75}$ This was supported by previous mobility studies of the PEO adsorbed on different sized polystyrene colloids which showed that PEO chain mobility was higher when the Pluronics were adsorbed on particles of higher curvature, indicating a decrease in the lateral interaction between the PEO chains. ${ }^{76}$ The decrease in liposome size most probably is due to a decrease in lateral interactions between the PEO chains of the adsorbed Pluronic molecules. ${ }^{75}$

Since our previous TEM experiments carried out on the DMPC-PFO system did not show any breakup of the vesicles with the formation of disk bilayers, ${ }^{3}$ we believe that in the DMPC : PFO system and, to a lesser extent, in DMPC : F, the reduction of the liposome size at high concentrations is compensated by the repulsion between the adsorbed compounds into the bilayer. Estimations were made of the changes of the number of fluorene molecules per liposome or PFO chains per liposome with the increase of the compound concentrations and are shown in Fig. 6B. Details are given elsewhere. ${ }^{3}$ (The number of liposomes formed was calculated from the liposomal radius and from the assumption of geometry parameters made by Huang and Mason.). ${ }^{77}$

Our results for DMPC : F are of the same order of magnitude of the calculations made by Schott et al. ${ }^{12}$ with various perylenes in S-PC. For liposomes with a diameter of about 70 to $80 \mathrm{~nm}$ about 200 to 300 dye molecules could be incorporated.

The concentrations of fluorophores per liposome is higher in the case of PFO than in the case of F. This could be related to their different structures (Fig. 1) and location in the DMPC bilayer as MD simulation shows (Fig. 3) and can explain the higher reduction of the DMPC liposome radius induced by PFO in comparison to $\mathrm{F}$.

\section{Photophysical properties of fluorenes in organic solvents and DMPC}

The absorption and emission spectra of the neutral fluorenes (F, TF, PFO and PFD) incorporated into DMPC liposomes were registered, and the normalized spectra are shown in Fig. 7A and $\mathrm{B}$, respectively. While it is possible that a small fraction of the DMPC system may be present as phospholipid bilayers rather 
than liposomes, this is unlikely to affect the overall photophysical behaviour. The main electronic spectroscopic properties of these compounds (absorption and emission maxima ( $\lambda_{\max }^{\mathrm{abs}}$ and $\lambda_{\max }^{\mathrm{em}}$ ), molar absorption coefficients $(\varepsilon)$ at the absorption maxima, and emission quantum yields $(\phi)$ ) enclosed in DMPC liposomes and in typical organic solvents (chloroform, cyclohexane and ethanol) are presented in Table 2. Their normalized absorption and emission spectra in chloroform, cyclohexane and ethanol are compared in Fig. S3 to S5 (ESI $\dagger$ ), while the effect of the environment on the spectra of each probe is shown separately in Fig. S6 to S9 (ESI†).

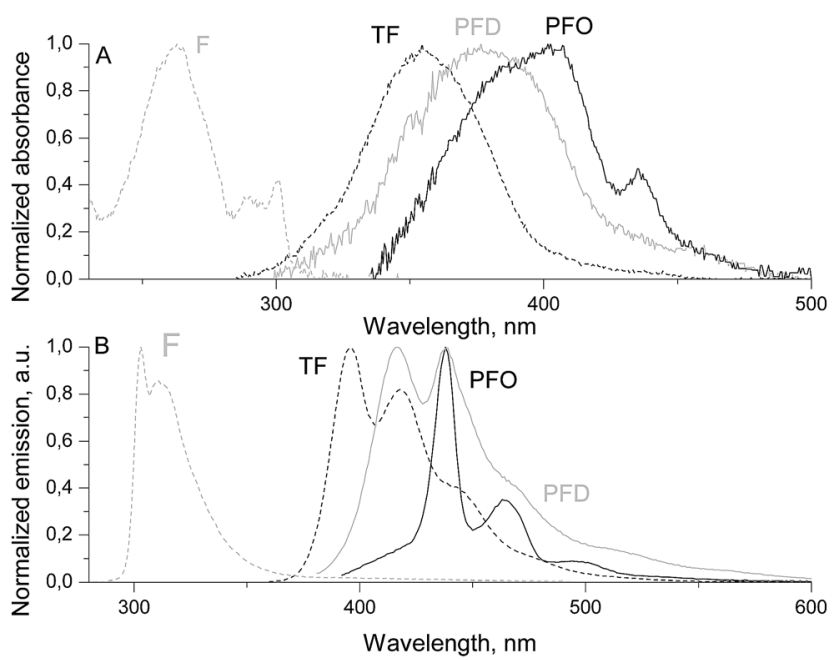

Fig. 7 (A) Normalized absorption spectra of $F\left(8.7 \times 10^{-6} \mathrm{M}\right.$, dashed gray line), TF $\left(8.3 \times 10^{-6} \mathrm{M}\right.$, dashed black line), PFO $\left(1.0 \times 10^{-5} \mathrm{M}\right.$, solid black line) and PFD $\left(1.1 \times 10^{-5} \mathrm{M}\right.$, solid gray line). (B) Normalized emission spectra of $F\left(9.7 \times 10^{-6} \mathrm{M}\right.$, dashed gray line $)$, TF $\left(8.3 \times 10^{-6}\right.$ $\mathrm{M}$, dashed black line), PFO $\left(5.8 \times 10^{-6} \mathrm{M}\right.$, solid black line $)$ and PFD $\left(6.95 \times 10^{-6} \mathrm{M}\right.$, solid gray line).
Although the absorption and emission spectra of $\mathrm{F}$ do not show mirror symmetry, they show a well resolved vibrational structures, in both organic solvents and the DMPC liposomes (Fig. 7 and S3 to S6 in the ESI $\dagger$ ). In contrast, as is common with fluorene based oligomers and polymers, the trimer and polymer absorption spectra in most of the organic solvents and TF and PFD in DPMC liposomes are broad and structureless and only the emission spectra show a well resolved vibrational structure (Fig. 7, and S7 to S9†). This is attributed to the fact that absorption is an instantaneous process which allows many possible segments with different conjugation lengths to absorb while only the lowest energy states emit. ${ }^{78}$ The high degree of conformational disorder in the oligomer and polymer implies an increase in the number of electronic states accessible and, as a consequence, the broadening of the absorption spectra and loss of the vibrational structure.

However, with PFO in DMPC, the absorption spectrum shows a vibrational structure with a new band at $438 \mathrm{~nm}$, which corresponds to the formation of the PFO $\beta$-phase (Fig. 7 and S8 $\dagger) .{ }^{3}$ Both the absorption and emission spectra are red-shifted for PFO in the DMPC bilayer compared with cyclohexane or chloroform. Moreover, from the comparison of the emission spectra of PFO in DMPC and ethanol (Fig. S8 $\dagger$ ), it can be concluded that a more planar PFO $\beta$-phase is formed in both ethanol and DMPC whereas, in chloroform and cyclohexane, a PFO $\alpha$-phase is predominant (Fig. S8 $\dagger$ ). It is well-known that the content of the PFO $\beta$-phase increases with the degree of aggregation in poor solvents such as a mixture of chloroform/ethanol with a content of ethanol above $40 \% .^{79}$ The macroscopic aggregates make the content of the $\beta$-phase much higher and stable. ${ }^{79}$

In contrast, with the absorption and emission spectra of PFD, there is no evidence for $\beta$-phase formation (Fig. S9†), confirming that the length of the alkyl chain substituent on the polyfluorene is one of the crucial factors which determines the formation of the $\beta$-phase when the polymer is enclosed in the

Table 2 Maxima of the absorption ( $\left.\lambda_{\max }^{\mathrm{abs}}\right)$ and emission $\left(\lambda_{\max }^{\mathrm{em}}\right)$ spectra, wavelength of the emission spectrum shoulders $\left(\lambda_{\mathrm{sh}}^{\mathrm{em}}\right)$, molar absorption coefficients at $\lambda_{\max }^{\mathrm{abs}}(\varepsilon)$ and emission quantum yield $(\phi)$. Molar concentrations for the oligomer and polymers are calculated in terms of the repeat units

\begin{tabular}{|c|c|c|c|c|c|c|c|}
\hline Solvent & Comp. & $\lambda_{\max }^{\mathrm{abs}}, \mathrm{nm}$ & $\varepsilon, \mathrm{mol}^{-1} \mathrm{~cm}^{-1} \mathrm{~L}$ & $\lambda_{\max }^{\mathrm{em}}, \mathrm{nm}$ & $\lambda_{\mathrm{sh} 1}^{\mathrm{em}}, \mathrm{nm}$ & $\lambda_{\mathrm{sh} 2}^{\mathrm{em}}, \mathrm{nm}$ & $\phi$ \\
\hline \multirow[t]{4}{*}{ Chloroform } & $\mathrm{F}$ & 265 & $18203 \pm 838$ & 305 & 314 & - & $0.029 \pm 0.003$ \\
\hline & $\mathrm{TF}$ & 352 & $25185 \pm 493$ & 394 & 415 & 441 & $0.82 \pm 0.07$ \\
\hline & PFD & 368 & $27210 \pm 172$ & 413 & 436 & 472 & $0.56 \pm 0.05$ \\
\hline & PFO & 386 & $15800 \pm 358$ & 415 & 441 & 473 & $0.59 \pm 0.06$ \\
\hline \multirow[t]{4}{*}{ Cyclohexane } & $\mathrm{F}$ & 262 & $14616 \pm 130$ & 302 & 309 & - & $0.68 \pm 0.07$ \\
\hline & $\mathrm{TF}$ & 350 & $21813 \pm 158$ & 390 & 411 & 435 & $0.97 \pm 0.01$ \\
\hline & PFD & 368 & $33349 \pm 467$ & 409 & 432 & 467 & $0.47 \pm 0.01$ \\
\hline & PFO & 383 & $15865 \pm 353$ & 410 & 436 & 465 & $0.52 \pm 0.09$ \\
\hline \multirow[t]{4}{*}{ Ethanol } & $\mathrm{F}$ & 262 & $14952 \pm 375$ & 302 & 309 & - & $0.73 \pm 0.02$ \\
\hline & $\mathrm{TF}$ & 346 & $23272 \pm 52$ & 390 & 410 & 435 & $0.88 \pm 0.06$ \\
\hline & PFD & 344 & $1479 \pm 11$ & 398 & 419 & & $0.70 \pm 0.01$ \\
\hline & PFO & - & - & 439 & 464 & 494 & - \\
\hline \multirow[t]{4}{*}{ DMPC } & $\mathrm{F}$ & 262 & $10284 \pm 410$ & 303 & 310 & - & $0.63 \pm 0.09$ \\
\hline & $\mathrm{TF}$ & 353 & $16939 \pm 1474$ & 396 & 418 & 444 & $0.70 \pm 0.09$ \\
\hline & PFD & 377 & $9030 \pm 1147$ & 417 & 438 & 467 & $0.23 \pm 0.04$ \\
\hline & PFO & 405 & $2920 \pm 330$ & 438 & 465 & 498 & $0.30 \pm 0.08$ \\
\hline
\end{tabular}


phospholipid bilayer. This is fully consistent with the effect of the side chain length on the behaviour of these polymers reported for methylcyclohexane. ${ }^{48}$ PFO has the optimal alkyl chain length for the formation of the $\beta$-phase because the side chain interactions are stable and sufficient to overcome steric repulsion and planarize the polymer backbone. Based on molecular dynamics and DFT simulations on PFO oligomers, and NMR and small angle neutron scattering results on polymer solutions over a wide concentration range, it has been shown that side-chain interactions play a major role in the association behaviour of this class of polymer. ${ }^{51}$

The $\beta$-phase is also not observed in the absorption and emission spectra of the trimer, regardless of the solvent considered or of the inclusion in DMPC liposomes (Fig. S7 in the ESI). $\dagger$ This indicates that the same factors that control the formation of the $\beta$-phase in solution are determinant for the formation of that phase when the fluorophore is enclosed in the phospholipid bilayer. The $\beta$-phase is formed by PFO only in poor solvents and at low temperatures when more than 9 repeat units constitute the polymer chain..$^{50}$

To test the effect of the structure and environment on the absorption and emission maxima, the spectra of different compounds in the same media are compared in Fig. 7, S3, S4 and S5 (in the ESI), $\dagger$ while the changes induced by the environment on the spectroscopic properties of a particular compound are shown in Fig. S6 to S9 (ESI). $\dagger$ The wavelength of the maxima of the absorption and emission spectra of these four compounds (F, TF, PFO and PFD) shows only modest shifts with the solvent polarity, as expected for typical $\pi \rightarrow \pi^{*}$ transitions.

However, a red shift of the absorption and emission maxima with the increase in conjugation of the probe is observed both in organic solvents and in DMPC-liposomes, following the expected order polymer $>$ trimer $>$ monomer, Table $2 .^{80}$ For the two polymers, there is also a red shift in absorption, and a smaller one in emission, with increasing molecular weight, $\mathrm{PFO}>\mathrm{PFD}$. In chloroform and cyclohexane, the red shift of the maximum of the absorption spectrum of TF with respect to the $\mathrm{F}$ is around $88 \mathrm{~nm}$ while those of PFD and PFO with respect to the trimer are around 17 and $33 \mathrm{~nm}$, respectively. Similar shifts are observed if the wavelengths of the emission maxima are considered (around $88 \mathrm{~nm}$ when TF is compared with $\mathrm{F}$ and around $20 \mathrm{~nm}$ when polymers are compared with the $\mathrm{TF}$ ). The difference in the spectral maxima between the monomer and the oligomer is much larger than between the oligomer to the polymer in both organic solvents and DMPC, which we believe justifies the use of the trimer in the MD simulations as a reasonable model of the polymer.

The shift between the maximum of absorption and emission (Stokes' shift) is around $42 \mathrm{~nm}$, and is relatively unaffected by the solvent, for F, TF and PFD in both DMPC and organic solvents (Table 2), indicating no significant differences in the photophysical behaviour of probes enclosed in liposomes or in the bulk organic solvent. A smaller Stokes' shift is seen with PFO (around $28 \mathrm{~nm}$ in chloroform and cyclohexane and $33 \mathrm{~nm}$ in DMPC).
The fluorescence quantum yields of F, TF, FPO and PFD (Table 2) in the organic solvents used are above 0.5, with the exception of $\mathrm{F}$ in chloroform. When data are available, such as for $\mathrm{F}^{81}$ in ethanol, ${ }^{82} \phi$ values obtained are in a good agreement with the literature values. The low value in chloroform is probably due to formation of contact charge transfer species, as has been reported for various other aromatics and chlorinated hydrocarbons. ${ }^{83}$

When the oligomers and polymers are enclosed in the phospholipid bilayer, the emission is quenched compared with the average values of $\phi$ in organic solvents. The reduction of the fluorescence quantum yield is lower for $\mathrm{TF}$ (about $20 \%$ ) than for the two polymers (PFD, $60 \%$ or PFO, $45 \%$ ). In addition, the absorption and emission spectra of the trimer and polymers are broader and red-shifted (more for PFO due to the formation of the $\beta$-phase) when enclosed in the DMPC bilayer compared with the spectra in organic solvents (Fig. S7, S8 and S9 in the ESI†). This suggests a strong inter-chain interaction, characteristic of aggregated conjugated polymers ${ }^{84}$ and is compatible with the fact that the chromophores are not homogeneously distributed within the liposomes (as in organic solvents), but they are confined within a reduced region of the phospholipid bilayer. The "real local" concentration will be higher than the average calculated from the total volume of the liposomes, leading to aggregated states which are more significant for oligomers and polymers due to the steric restrictions imposed by their more bulky and rigid structures.

The attribution of the stronger quenching of the emission of PFO (60\%) compared with $\mathrm{F}(10 \%)$ to aggregation is in good agreement with the results of dynamic light scattering (Fig. 6), which showed a higher concentration of fluorophore per liposome for PFO.

Time-resolved luminescence provides further details on these interactions. In Table 3, the decay times $\left(\tau_{\mathrm{i}}\right)$, pre-exponential factors $\left(a_{\mathrm{i}}\right)$ and chi-squared values $\left(\chi^{2}\right)$ of F, TF, PFD and PFO in several solvents and DMPC are shown, together with the excitation and emission wavelengths used. For DMPC, average values based on the values obtained at several fluorophore concentrations are shown. The details are given in Tables S1 to S4 in the ESI. $\dagger$

Fluorene shows monoexponential decays in organic solvents while these become biexponential when enclosed in the DMPC bilayer, with one lifetime close to that in organic solvents $(\approx 1 \mathrm{~ns})$ and a second one markedly longer ( $3.2 \mathrm{~ns})$. From Table 2 , there is a very modest decrease in the fluorescence quantum yield, and a more marked decrease in the molar absorption coefficient of $\mathrm{F}$ in DMPC compared with cyclohexane. From the Strickler-Berg relationship ${ }^{85}$ the decrease in $\varepsilon$ may reflect an increase in radiative lifetime. The contribution to the total emission of each component $\left(f_{\mathrm{i}}\right)$ can be calculated as $f_{\mathrm{i}}=\left(a_{\mathrm{i}} \tau_{\mathrm{i}}\right) /$ $\left(\Sigma_{\mathrm{i}}\left(a_{\mathrm{i}} \tau_{\mathrm{i}}\right){ }^{80}{ }^{80}\right.$ For fluorene, the average contribution to the total emission of the species with a lifetime of $1160 \mathrm{ps}$ is $26 \%$ and that one with lifetime of $3220 \mathrm{ps}$ is around $74 \%$. These results are compatible with the migration of $\mathrm{F}$ into the bilayer described in the MD simulation section (Fig. 2B and C). From this model and the lifetime data, it is feasible that $3 / 4$ of the fluorene emission (lifetime $3220 \mathrm{ps}$ ) is due to molecules which 
Table 3 Decay times $\left(\tau_{i}\right)$, pre-exponential factors $\left(a_{i}\right)$, and chi-squared $\left(\chi^{2}\right)$ of F, TF, PFD and PFO in several solvents and DMPC. For DMPC, average values based on the values obtained at several fluorophore concentrations are shown. The details are given in Tables S1 to S4 in the ESI. Excitation wavelength $\left(\lambda_{\text {exc }}\right)$ and emission wavelength $\left(\lambda_{\text {em }}\right)$

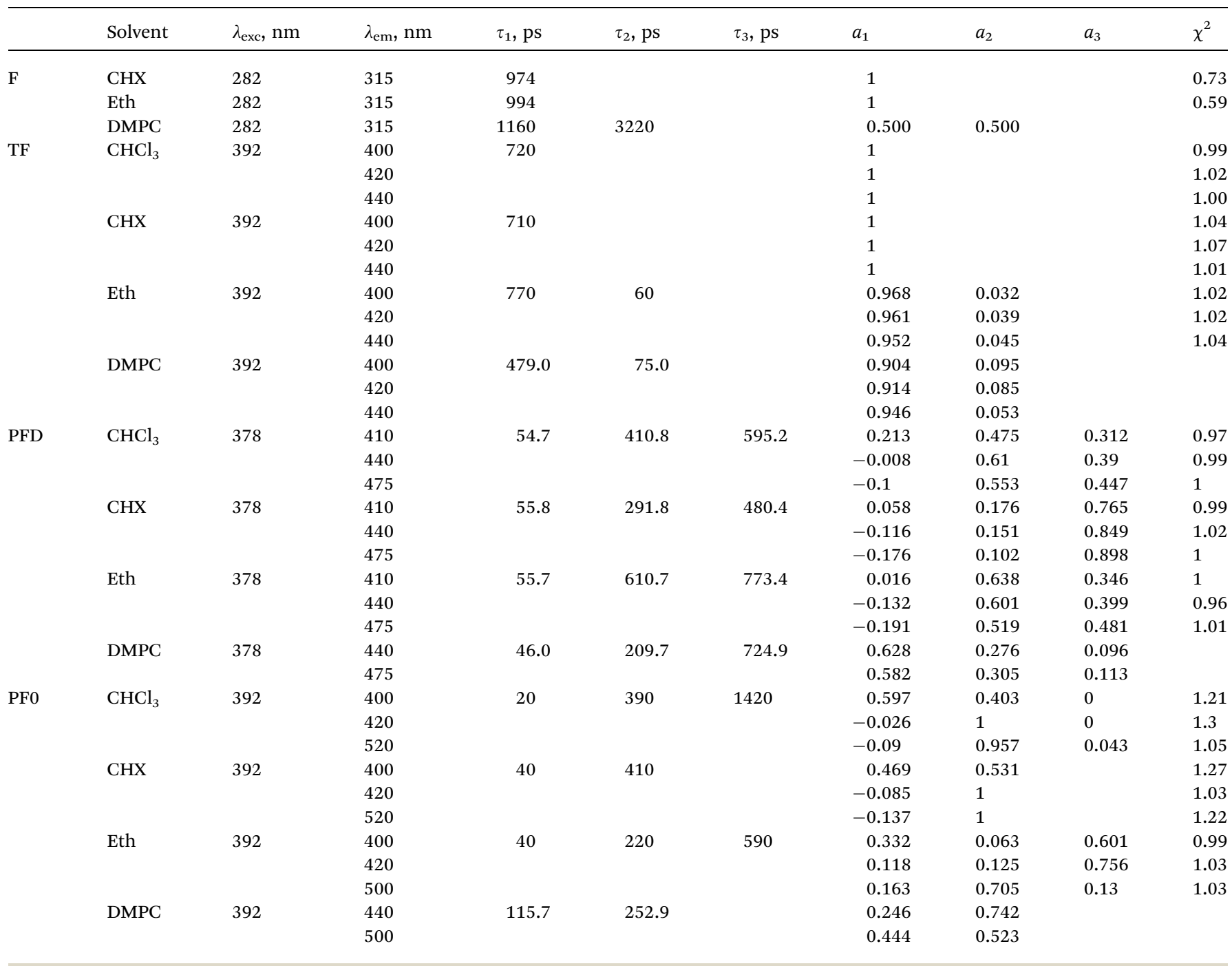

are close to the head groups of the phospholipid in a more restricted environment, while the rest of the emission comes from molecules near the centre of the bilayer, where the polarity is similar to that of cyclohexane. Moreover, the contribution to the total emission of the fluorene with a lifetime of 1160 ps increases from $16 \%$ to $30 \%$ upon increasing the fluorene concentration from around $10^{-6} \mathrm{M}$ to $10^{-5} \mathrm{M}$, indicating partitioning of the probe from the head group region to the centre of the bilayer.

To a good approximation, the decays of the trimer TF can be also considered monoexponential in both organic solvents and liposomes because the contribution to the total emission of the short component is less than $1 \%$ for TF in ethanol (60 ps) and less than $2 \%$ for TF in DMPC (75 ps) showing only residual importance. In addition TF lifetime is not dependent on the concentration of TF within the liposome. The monoexponential decays are compatible with only one environment for TF in very good agreement with the MD simulation results, where it is in the centre of the bilayer (Fig. 3), parallel to the leaflet of the bilayer (Fig. 2D) with a very slow diffusion (Fig. 2C). The explanation for the decrease of TF quantum yield (Table 2) and lifetime (Table 3) when TF is in the phospholipid bilayer is not yet clear, but appears to be associated with some enhanced nonradiative decay pathway. The appearance of the short component, although only with a minor contribution in ethanol and DMPC, could be related to energy migration between adjacent trimers.

In contrast, PFD and PFO show the characteristic complex decays commonly seen with polyfluorenes. ${ }^{78}$ For PFD, tri-exponential decays are observed both in the organic solvents and enclosed in DMPC liposomes. The shortest component of around 55 ps in the organic solvents (Table 3), with a contribution of less than $5 \%$ to the total emission, appears as a rise time (negative amplitude) when the decay is collected in the emission tails (440 and $475 \mathrm{~nm}$ ). This is the signature of fast conformation relaxations of the PFD backbone ${ }^{86,87}$ and is not observed for PFD within the phospholipid bilayer, which indicates that PFD is in a more rigid environment in the liposomes. 
For most of the systems, the intermediate and the longest components show similar contributions to the total emission, except for PFD in cyclohexane which has a predominant contribution of the longest component (480.4 ps).

PFO shows multiexponential decays in chloroform and cyclohexane (Table 3). In these solvents, the $\beta$-phase is not formed (Fig. S8 in the ESI $\dagger$ ) and the shortest component of the decays has a rise time when the emission is collected at 420 and $520 \mathrm{~nm}$ indicating again a fast conformation relaxation of the PFO backbone ${ }^{78}$ which is not observed when the $\beta$-phase is formed, either in ethanol or DMPC (Fig. S8 $†$ ).

The characteristic biexponential decays of PFO in DMPC (Tables 3 and $\mathrm{S} 4 \dagger$ ) with average lifetimes around 116 and 253 are the signature of the formation of the $\beta$-phase as has previously been discussed. ${ }^{3}$

PFO in ethanol shows intermediate behaviour between that observed in better solvents (chloroform and cyclohexane), with a predominance of the contribution of the longest component (590 ps, above 90\%) when the emission is registered in the main emission band (400 and $420 \mathrm{~nm}$ ) and the prevalence of the intermediate band $(220 \mathrm{ps})$, characteristic of the $\beta$-phase formation, when emission is registered at $500 \mathrm{~nm}$. This indicates that PFO forms the $\beta$-phase in ethanol, as indicated in the steady-state study (Fig. S8 $\dagger$ ), but to a lesser extent than when is in DMPC liposomes.

\section{Conclusions}

The photophysics of neutral fluorenes: F, TF, PFO and PFD has been studied with these fluorescent probes incorporated into the bilayer of aqueous suspensions of DMPC liposomes. With PFO, the formation of the $\beta$-phase was observed. However, with its homologous TF (oligomer) and PFD (polymer with a longer substituent alkyl chains) this is not observed, indicating both that a minimum number of backbone repeat units and the octyl chain as a substituent of the 9-carbon atom in the fluorene group are needed to form the $\beta$-phase efficiently in liposomes. The same factors are observed in organic solvents, showing that the polymer-phospholipid interaction does not allow us to overcome these requirements for the formation of the $\beta$-phase.

MD simulations show that all these compounds are incorporated into the phospholipid bilayer. However, while the small molecules B, BP and F are orientated parallel to the phospholipid alkyl chains (and perpendicular and close to the interface), the trimer and polymers, are located in the centre of the bilayer parallel to the plane of the head groups and with the alkyl chain substituents orientated towards the phospholipid alkyl chains. This result perfectly matches with the decrease of the DMPC IR band at $1467 \mathrm{~cm}^{-1}$ assigned to the non-interdigitated part of the DMPC alkyl chains when deuterated PFO is incorporated into the phospholipid bilayer. The partial interdigitation of the phospholipid and polymer alkyl chains is suggested to be partly responsible for the increase of the transition temperature of DMPC upon addition of PFO and PFD as seen by DSC, while F induces a decrease of the transition temperature and TF shows an intermediate behaviour.
The diffusion of these molecules in the phospholipid bilayer follows the order $\mathrm{B}>\mathrm{BP}>\mathrm{F}>\mathrm{TF}$, as expected from their molecular sizes.

According to the alkyl chain order parameter, $S_{\mathrm{CD}}, \mathrm{F}$ and $\mathrm{BP}$ increase the organization in the hydrophobic part of the bilayer towards the interface but with $\mathrm{F}$ these effects are extended towards the interior of the bilayer. However, it seems that it is the order induced in the centre of the bilayer by the incorporation of polymers (particularly at the end of the phospholipid alkyl chains) through the interdigitation with the polymer substituent which determines the increase of the DMPC phase transition temperature.

\section{Acknowledgements}

We are grateful to Professor U. Scherf and Dr A. T. Marques for the gift of the alkyl chain deuterated PFO- $\mathrm{d}_{34}$ used in IR spectral studies. MEC and FEDER are thanked for financial support through the MAT2008-06079/MAT. Funding from the European Community's Seventh Framework Programme under grant agreement no. 228334 is acknowledged. Financial support of the Coimbra Chemistry Centre from the FCT through project PEst-OE/QUI/UI0313/2014 is gratefully acknowledged.

\section{References}

1 G. Gregoriadis and Y. Perrie, in Encyclopedia of Life Sciences, John Wiley \& Sons, Ltd, 2010, pp. 1-8.

2 V. Librando, M. G. Sarpietro and F. Castelli, Environ. Toxicol. Pharmacol., 2003, 14, 25-32.

3 M. J. Tapia, M. Monteserín, H. D. Burrows, J. S. Seixas de Melo, J. Pina, R. A. E. Castro, S. García and J. Estelrich, J. Phys. Chem. B, 2011, 115, 5794-5800.

4 R. Mallavia, M. J. Martínez-Tomé, R. Vázquez-Guilló, Z. Kahveci, A. Estepa and C. R. Mateo, ACS Appl. Mater. Interfaces, 2013, 5, 2952-2958.

5 J. Sabín, G. Prieto, J. Estelrich, F. Sarmiento and M. Costas, J. Colloid Interface Sci., 2010, 348, 388-392.

6 T. Ishøy and K. Mortensen, Langmuir, 2005, 21, 1766-1775.

7 S. Vemuri and C. T. Rhodes, Pharm. Acta Helv., 1995, 70, 95111.

8 S. Prabhu, M. Ortega and C. Ma, Int. J. Pharm., 2005, 301, 209-216.

9 V. P. Torchilin, Nat. Rev. Drug Discovery, 2005, 4, 145-160.

10 A. S. L. Derycke and P. A. M. de Witte, Adv. Drug Delivery Rev., 2004, 56, 17-30.

11 J.-P. Starck, Y. Nakatani, G. Ourisson, D. J. Cowley and G. Duportail, New J. Chem., 1996, 20, 1293-1299.

12 H. Schott, D. V. Cunow and H. Langhals, Biochim. Biophys. Acta, 1992, 1110, 151-157.

13 K. Kachel, E. Asuncion-Punzalan and E. London, Biochim. Biophys. Acta, 1998, 1374, 63-76.

14 T. Parasassi, G. De Stasio, R. M. Rusch and E. Gratton, Biophys. J., 1991, 59, 466-475.

15 E. Kalb, F. Paltauf and A. Hermetter, Biophys. J., 1989, 56, 1245-1253. 
16 C. R. Mateo, J.-C. Brochon, M. P. Lillo and A. U. Acuña, Biophys. J., 1993, 65, 2237-2247.

17 A. K. Lala, R. R. Dixit, V. Koppaka and S. Patel, Biochemistry, 1988, 27, 8981-8989.

18 A. K. Lala and V. Koppaka, Biochemistry, 1992, 31, 55865593.

19 E. D. Matayoshi and A. M. Kleinfeld, Biophys. J., 1981, 35, 215-235.

20 Z. Aihua, G. Qiang, H. Quanguo, G. Rong and Y. Chunwei, Colloids Surf., A, 2003, 224, 75-82.

21 A. T. Ngo and G. Cosa, Langmuir, 2010, 26, 6746-6754.

22 A. T. Ngo, P. Karam, E. Fuller, M. Burger and G. Cosa, J. Am. Chem. Soc., 2008, 130, 457-459.

23 P. Karam, A. T. Ngo, I. Rouiller and G. Cosa, Proc. Natl. Acad. Sci. U. S. A., 2010, 107, 17480-17485.

24 Z. Kahveci, M. Martínez-Tomé, R. Esquembre, R. Mallavia and C. Mateo, Materials, 2014, 7, 2120-2140.

25 Z. Kahveci, M. J. Martínez-Tomé, R. Mallavia and C. R. Mateo, Biomacromolecules, 2013, 14, 1990-1998.

26 A. J. Heeger, Chem. Soc. Rev., 2010, 39, 2354-2371.

27 A. Duarte, K.-Y. Pu, B. Liu and G. C. Bazan, Chem. Mater., 2010, 23, 501-515.

28 F. Huang, H. Wu and Y. Cao, Chem. Soc. Rev., 2010, 39, 25002521.

29 D. H. Park, M. S. Kim and J. Joo, Chem. Soc. Rev., 2010, 39, 2439-2452.

30 Q. Wang and D. Ma, Chem. Soc. Rev., 2010, 39, 2387-2398.

31 J. Li and A. C. Grimsdale, Chem. Soc. Rev., 2010, 39, 23992410.

32 H. Klauk, Chem. Soc. Rev., 2010, 39, 2643-2666.

33 A. T. Ngo, P. Karam and G. Cosa, Pure Appl. Chem., 2011, 83, 43-55.

34 S. Chemburu, E. Ji, Y. Casana, Y. Wu, T. Buranda, K. S. Schanze, G. P. Lopez and D. G. Whitten, J. Phys. Chem. B, 2008, 112, 14492-14499.

35 Y. Liu, K. Ogawa and K. S. Schanze, Anal. Chem., 2008, 80, 150-158.

36 L. Ding, E. Y. Chi, K. S. Schanze, G. P. Lopez and D. G. Whitten, Langmuir, 2009, 26, 5544-5550.

37 L. Ding, E. Y. Chi, S. Chemburu, E. Ji, K. S. Schanze, G. P. Lopez and D. G. Whitten, Langmuir, 2009, 25, 1374213751.

38 Y. Wang, E. M. Jones, Y. Tang, E. Ji, G. P. Lopez, E. Y. Chi, K. S. Schanze and D. G. Whitten, Langmuir, 2011, 27, 10770-10775.

39 P. Howes, M. Green, J. Levitt, K. Suhling and M. Hughes, J. Am. Chem. Soc., 2010, 132, 3989-3996.

40 P. Howes, M. Green, A. Bowers, D. Parker, G. Varma, M. Kallumadil, M. Hughes, A. Warley, A. Brain and R. Botnar, J. Am. Chem. Soc., 2010, 132, 9833-9842.

41 S. Kolusheva, T. Shahal and R. Jelinek, J. Am. Chem. Soc., 2000, 122, 776-780.

42 M. J. Winokur, J. Slinker and D. L. Huber, Phys. Rev. B: Condens. Matter Mater. Phys., 2003, 67, 184106.

43 F. B. Dias, J. Morgado, A. L. Maçanita, F. P. da Costa, H. D. Burrows and A. P. Monkman, Macromolecules, 2006, 39, 5854-5864.
44 L. Huang, L. Zhang, X. Huang, T. Li, B. Liu and D. Lu, J. Phys. Chem. B, 2014, 118, 791-799.

45 M. J. Tapia, M. Monteserín, J. Pina, J. S. Seixas de Melo and H. D. Burrows, J. Colloid Sci. Biotechnol., 2013, 243-248.

46 M. J. Tapia, M. Monteserín, H. D. Burrows, J. S. Seixas de Melo and J. Estelrich, Photochem. Photobiol., 2013, 14711478.

47 C. W. Cone, R. R. Cheng, D. E. Makarov and D. A. Vanden Bout, J. Phys. Chem. B, 2011, 115, 12380-12385.

48 D. W. Bright, F. B. Dias, F. Galbrecht, U. Scherf and A. P. Monkman, Adv. Funct. Mater., 2009, 19, 67-73.

49 D. W. Bright, F. Galbrecht, U. Scherf and A. P. Monkman, Macromolecules, 2010, 43, 7860-7863.

50 E. Da Como, E. Scheler, P. Strohriegl, J. Lupton and J. Feldmann, Appl. Phys. A: Mater. Sci. Process., 2009, 95, 61-66.

51 L. L. G. Justino, M. L. Ramos, M. Knaapila, A. T. Marques, C. J. Kudla, U. Scherf, L. Almásy, R. Schweins, H. D. Burrows and A. P. Monkman, Macromolecules, 2011, 44, 334-343.

52 M. M. Lapinski, A. Castro-Forero, A. J. Greiner, R. Y. Ofoli and G. J. Blanchard, Langmuir, 2007, 23, 11677-11683.

53 K. Suzuki, A. Kobayashi, S. Kaneko, K. Takehira, T. Yoshihara, H. Ishida, Y. Shiina, S. Oishi and S. Tobita, Phys. Chem. Chem. Phys., 2009, 11, 9850-9860.

54 D. F. Eaton, Pure Appl. Chem., 1988, 60, 1107-1114.

55 J. Pina, J. Seixas de Melo, H. D. Burrows, A. L. Maçanita, F. Galbrecht, T. Bünnagel and U. Scherf, Macromolecules, 2009, 42, 1710-1719.

56 G. Striker, V. Subramaniam, C. A. M. Seidel and A. J. Volkmer, J. Phys. Chem. B, 1999, 103, 8612-8617.

57 K. Sonogashira, Y. Tohda and N. Hagihara, Tetrahedron Lett., 1975, 16, 4467-4470.

58 A. W. Schuttelkopf and D. M. F. Van Aalten, Acta Crystallogr., Sect. D: Biol. Crystallogr., 2004, 60, 1355-1363.

59 L. D. Schuler, X. Daura and W. F. Van Gunsteren, J. Comput. Chem., 2001, 22, 1205-1218.

60 A. Kukol, J. Chem. Theory Comput., 2009, 5, 615-626.

61 B. Hess, C. Kutzner, D. Van der Spoel and E. Lindahl, J. Chem. Theory Comput., 2008, 4, 435-447.

62 B. Hess, H. Bekker, H. J. C. Berendsen and J. G. E. M. Fraaije, J. Comput. Chem., 1997, 18, 1463-1472.

63 W. Humphrey, A. Dalke and K. Schulten, J. Mol. Graphics, 1996, 14, 33-38.

64 J. A. S. Almeida, E. F. Marques, A. S. Jurado and A. A. C. C. Pais, Phys. Chem. Chem. Phys., 2010, 12, 1446214476.

65 J. Čurdová, P. Čapková, J. Plášek, J. Repáková and I. Vattulainen, J. Phys. Chem. B, 2007, 111, 3640-3650.

66 L. M. S. Loura, A. M. T. M. do Canto and J. Martins, Biochim. Biophys. Acta, Biomembr., 2013, 1828, 1094-1101.

67 W. K. Nitschke, C. C. Vequi-Suplicy, K. Coutinho and H. Stassen, J. Phys. Chem. B, 2012, 116, 2713-2721.

68 A. Kyrychenko and J. Waluk, Biophys. Chem., 2008, 136, 128135.

69 C.-J. Högberg, A. Maliniak and A. P. Lyubartsev, Biophys. Chem., 2007, 125, 416-424. 
70 H.-S. Wang, Y.-H. Yang and Y. Ozaki, Spectrosc. Spectral Anal., 2010, 30, 892-896.

71 F. Castelli, D. Micieli, S. Ottimo, Z. Minniti, M. G. Sarpietro and V. Librando, Chemosphere, 2008, 73, 1108-1114.

72 J. A. S. Almeida, S. P. R. Pinto, Y. Wang, E. F. Marques and A. A. C. C. Pais, Phys. Chem. Chem. Phys., 2011, 13, 1377213782 .

73 J. A. S. Almeida, H. Faneca, R. A. Carvalho, E. F. Marques and A. A. C. C. Pais, PLoS One, 2011, 6, 1-12.

74 Y. Y. Chieng and S. B. Chen, J. Phys. Chem., 2009, 113, 1493414942.

75 M. Johnsson, M. Silvander, G. Karlsson and K. Edwards, Langmuir, 1999, 15, 6314-6325.

76 J.-T. Li, K. D. Caldwell and N. Rapoport, Langmuir, 1994, 10, 4475-4482.

77 C. Huang and J. T. Mason, Proc. Natl. Acad. Sci. U. S. A., 1978, 75, 308-310.

78 A. P. Monkman, C. Rothe, S. King and F. Dias, Adv. Polym. Sci., 2008, 212, 187-225.
79 L. Huang, X. Huang, G. Sun, C. Gu, D. Lu and Y. Ma, J. Phys. Chem. C, 2012, 116, 7993-7999.

80 J. R. Lakowicz, Principles of Fluorescence Spectroscopy, Kluwer Academic, Plenum Publishers, New York, 2004.

81 CRC Handbook of Organic Photochemistry, CRC Press, Inc., 1989.

82 E. R. Pantke and H. Labhart, Chem. Phys. Lett., 1972, 16, 255259.

83 K. M. C. Davis and M. F. Farmer, J. Chem. Soc. B, 1968, 859862.

84 C. Y. Tan, M. R. Pinto and K. S. Schanze, Chem. Commun., 2002, 446-447.

85 S. J. Strickler and R. A. Berg, J. Chem. Phys., 1962, 37, 814822.

86 F. B. Dias, A. L. Maçanita, J. Seixas de Melo, H. D. Burrows, R. Güntner, U. Scherf and A. P. Monkman, J. Chem. Phys., 2003, 118, 7119-7126.

87 J. Pina, J. S. Seixas de Melo, N. Koenen and U. Scherf, J. Phys. Chem. B, 2013, 117, 7370-7380. 